\title{
The effect of cross-sex hormonal treatment on gender dysphoria individuals' mental health: a systematic review
}

This article was published in the following Dove Press journal:

Neuropsychiatric Disease and Treatment

4 August 2016

Number of times this article has been viewed

\author{
Rosalia Costa' \\ Marco Colizzi ${ }^{2}$ \\ 'Gender Identity Development \\ Service, Tavistock and Portman NHS \\ Foundation Trust, Tavistock Centre, \\ ${ }^{2}$ Department of Psychosis Studies, \\ Institute of Psychiatry, Psychology \& \\ Neuroscience, King's College London, \\ London, UK
}

\begin{abstract}
Cross-sex hormonal treatment represents a main aspect of gender dysphoria health care pathway. However, it is still debated whether this intervention translates into a better mental well-being for the individual and which mechanisms may underlie this association. Although sex reassignment surgery has been the subject of extensive investigation, few studies have specifically focused on hormonal treatment in recent years. Here, we systematically review all studies examining the effect of cross-sex hormonal treatment on mental health and well-being in gender dysphoria. Research tends to support the evidence that hormone therapy reduces symptoms of anxiety and dissociation, lowering perceived and social distress and improving quality of life and self-esteem in both male-to-female and female-to-male individuals. Instead, compared to female-to-male individuals, hormone-treated male-to-female individuals seem to benefit more in terms of a reduction in their body uneasiness and personality-related psychopathology and an amelioration of their emotional functioning. Less consistent findings support an association between hormonal treatment and other mental health-related dimensions. In particular, depression, global psychopathology, and psychosocial functioning difficulties appear to reduce only in some studies, while others do not suggest any improvement in these domains. Results from longitudinal studies support more consistently the association between hormonal treatment and improved mental health. On the contrary, a number of cross-sectional studies do not support this evidence. This review provides possible biological explanation vs psychological explanation (direct effect vs indirect effect) for the hormonal treatment-induced better mental well-being. In conclusion, this review indicates that gender dysphoria-related mental distress may benefit from hormonal treatment intervention, suggesting a transient reaction to the nonsatisfaction connected to the incongruent body image rather than a stable psychiatric comorbidity. In this perspective, timely hormonal treatment intervention represents a crucial issue in gender dysphoria individuals' mental health-related outcome.
\end{abstract}

Keywords: estrogen, testosterone, transsexualism, psychiatry, psychosocial wellbeing

\section{Introduction}

A strong and persistent cross-gender identification as well as a persistent discomfort with one's natal sex or a sense of inappropriateness in the gender role of that sex has been considered as a core aspect of nonconforming gender identity since the first inclusion of the condition in the Diagnostic and Statistical Manual of Mental Disorders (DSM) of the American Psychiatric Association. ${ }^{1}$ Until the last DSM revision, ${ }^{2}$ the condition has been known as "transsexualism/gender identity disorder". ${ }^{1,3,4}$ In 2013, the American Psychiatric Association has significantly revised the condition definition in an attempt to balance between the competing issues of depathologizing nonconforming
Correspondence: Marco Colizzi Department of Psychosis Studies, Institute of Psychiatry, Psychology \& Neuroscience, King's College London, 16 De Crespigny Park, London SE5 8AF, UK

Tel +442078480049

Fax +442078480976

Email marco.v.colizzi@kcl.ac.uk 
gender identity vs access to care. ${ }^{2,5}$ As a consequence, the condition has been renamed "gender dysphoria" in order to imply a diagnostic entity in its own right, not necessarily associated with severe comorbid psychiatric findings, and better characterize the gender incongruence-related experience and discomfort. ${ }^{2}$ However, psychiatric comorbidity among individuals with gender dysphoria is still a matter of discussion. Some studies have reported a high prevalence of major psychiatric disorders in gender dysphoria.$^{6-8}$ Instead, other research suggests a low level of psychopathology. ${ }^{9-11} \mathrm{~A}$ recent review has concluded that gender dysphoria individuals appear to have a higher risk of psychiatric comorbidity. ${ }^{12}$ However, as reported by the authors, many studies were methodologically weak, and sex reassignment procedures appeared to be beneficial in reducing mental distress. ${ }^{12}$ Therefore, the lack of consistency between studies about gender dysphoria individuals' psychiatric comorbidity could be partially explained by their design, which usually did not explore the role of the lack of treatment in gender dysphoria individuals' mental distress.

For most gender dysphoria individuals, the subjective experience of persistent cross-gender identification and discomfort with their natal sex may be a stressful situation and cause clinical distress or impairment in important areas of functioning. ${ }^{9} 13,14$ Suffering from severe gender incongruence usually leads individuals to pursue hormone treatment and sex reassignment surgery. The purpose of these sex reassignment procedures is to alleviate the individual's distress by reducing the discrepancy between the individuals' biological sex and their experience. ${ }^{15}$ Over the last few decades, research has prevalently focused on the effect of sex reassignment surgery on gender dysphoria individuals' mental health. In particular, previous research has suggested that sex reassignment surgery improves gender dysphoria individuals' well-being, increasing their personal and general satisfaction, ${ }^{16-18}$ selfconfidence with body image, ${ }^{19-22}$ and quality of life. ${ }^{23-26} \mathrm{In}$ contrast, the role of the cross-sex hormonal treatment in the well-being of gender dysphoria individuals has been the subject of relatively little investigation. A 2010 meta-analysis has identified 28 observational studies of the effect of sex reassignment on gender dysphoria individuals' well-being. ${ }^{27}$ However, only five studies specifically examined the impact of hormonal treatment and two of them focused on genderrelated cognitive aspects, which could be influenced by crosssex hormones and per se do not inform on hormone-treated individuals' mental health or well-being. ${ }^{28,29}$ The remaining three studies addressed the effect of hormonal treatment on gender dysphoria individuals' psychological profile, ${ }^{30}$ emotional repercussions, ${ }^{31}$ and transformation satisfaction. ${ }^{32}$
However, due to its design, the latter one ${ }^{32}$ could not infer an association between hormonal treatment and better transformation satisfaction.

Studying the effect of cross-sex hormonal treatment on mental health in gender dysphoria may help to disentangle the burden of psychiatric comorbidity among individuals suffering from this condition. In recent years, a larger body of evidence has accumulated from studies on the specific effect of hormonal treatment on different mental health-related outcome measures. The purpose of this review is to bring together and discuss all available data generated by crosssectional and longitudinal studies that have investigated the effect of hormone therapy on mental health by carrying out a systematic literature search for all such data.

\section{Materials and methods Inclusion/exclusion criteria}

Inclusion criteria for studies were as follows: 1) studies in gender dysphoria, 2) studies investigating the mid/ long-term effects of cross-sex hormonal treatment, and 3) studies measuring mental health-related parameters, including a) depression, b) anxiety, c) personality, d) quality of life, e) dissociative symptoms, f) psychopathology, g) psychosocial/emotional functioning, h) distress, and i) self-esteem. Exclusion criteria were as follows: 1) studies in which cross-sex hormonal treatment was not the intervention of interest (ie, studies including cross-sex hormonal treatment only as a confounder/covariate of no interest) and 2) studies in which the mental health-related outcomes were not directly reported upon.

\section{Search strategy}

A final search was undertaken on May 16, 2016. The search terms used were as follows: (gender dysphoria or gender identity disorder or transsexualism) and (hormon* or crosssex hormonal treatment or hormone therapy) and (psych* or psychiatry or psychopathology or mental health or anxiety or depression). This search was undertaken in Medline, EMBASE, and PsycINFO using the OvidSP platform. All studies published in any language and indexed in the above databases were included. Reference lists from all identified relevant studies, reviews, and conference abstracts were screened for any additional relevant studies.

\section{Data extraction}

Demographic and methodological variables and outcome data for studies identified were extracted into a spreadsheet. Primary outcomes of interest were mental health-related parameters. These were compared within hormone-treated 
cohorts and/or between hormone-treated and nonhormonetreated control groups.

\section{Risk of bias}

Risk of bias and quality assessment of the methodologically heterogeneous group of studies (Table 1) reviewed here required a suitably inclusive and flexible approach. For this purpose, an adapted set of criteria, suggested by the Agency for Healthcare Research and Quality guidance, ${ }^{33}$ amended as appropriate for observational studies in humans were used (Table 2). Risk of systematic bias across studies was further identified by assessing all articles for possible confounding factors, such as study population definition, mental health comorbidity, attrition, comparability of subjects, cross-sex hormonal treatment duration, type, and dosage (Table 3).

\section{Results Study selection}

In total, 680 records were identified. All abstracts of the records were screened against the inclusion and exclusion criteria. A final list of 17 studies was identified for systematic analysis in this review. Cumulatively, the included studies investigated different aspects of mental health (Table 1) in response to cross-sex hormonal treatment, using both independent-groups (cross-sectional studies) and repeated measures (longitudinal studies) designs. These include 1) depression, mood states, and self-esteem; 2) anxiety and tension; 3) global and personality-related psychopathology; 4) mental health-related quality of life; 5) dissociative symptoms; 6) psychosocial, interpersonal, and emotional functioning; and 7) social distress, perceived stress,

Table I Studies included in the review

\begin{tabular}{|c|c|c|c|}
\hline Study & Type of study & Target population(s) & Mental health parameters \\
\hline Bonierbale et $\mathrm{al}^{42}$ & Cross-sectional study & $\begin{array}{l}\text { GID adults with CSHT vs GID adults } \\
\text { without CSHT }\end{array}$ & Personality-related psychopathology \\
\hline Bouman et $\mathrm{al}^{38}$ & Cross-sectional study & $\begin{array}{l}\text { MtF adults with CSHT vs MtF adults } \\
\text { without CSHT }\end{array}$ & $\begin{array}{l}\text { Anxiety, depression, self-esteem, and } \\
\text { interpersonal functioning }\end{array}$ \\
\hline Colizzi et al $^{45}$ & Longitudinal study & GD adults before and after CSHT & Dissociative symptoms \\
\hline Colizzi et $\mathrm{al}^{37}$ & Longitudinal study & GID adults before and after CSHT & $\begin{array}{l}\text { Anxiety, depression, psychopathological } \\
\text { symptoms, and psychosocial functioning }\end{array}$ \\
\hline Fisher et $\mathrm{al}^{39}$ & Cross-sectional study & $\begin{array}{l}\text { GD adults with CSHT vs GD adults } \\
\text { without CSHT }\end{array}$ & $\begin{array}{l}\text { Body uneasiness and } \\
\text { psychopathological symptoms }\end{array}$ \\
\hline Gómez-Gil et al ${ }^{44}$ & Cross-sectional study & $\begin{array}{l}\text { GID adults with CSHT vs GID adults } \\
\text { without CSHT }\end{array}$ & Mental health-related quality of life \\
\hline Heylens et $\mathrm{al}^{40}$ & Longitudinal study & GID adults before and after CSHT & Psychopathological symptoms \\
\hline Colizzi et $\mathrm{al}^{14}$ & Longitudinal study & GID adults before and after CSHT & Perceived stress \\
\hline Gorin-Lazard et $\mathrm{al}^{36}$ & Cross-sectional study & $\begin{array}{l}\text { GID adults with CSHT vs GID adults } \\
\text { without CSHT }\end{array}$ & $\begin{array}{l}\text { Self-esteem, depression, quality of life, } \\
\text { and psychosocial functioning }\end{array}$ \\
\hline Gómez-Gil et $\mathrm{al}^{35}$ & Cross-sectional study & $\begin{array}{l}\text { GID adults with CSHT vs GID adults } \\
\text { without CSHT }\end{array}$ & Social distress, anxiety, and depression \\
\hline Gorin-Lazard et al ${ }^{43}$ & Cross-sectional study & $\begin{array}{l}\text { GID adults with CSHT vs GID adults } \\
\text { without CSHT }\end{array}$ & Mental health-related quality of life \\
\hline Gómez-Gil et $\mathrm{al}^{41}$ & Cross-sectional study & $\begin{array}{l}\text { GID adults with CSHT vs GID adults } \\
\text { without CSHT }\end{array}$ & Personality-related psychopathology \\
\hline Miles et $\mathrm{al}^{29}$ & $\begin{array}{l}\text { I. Longitudinal study } \\
\text { 2. Longitudinal study } \\
\text { 3. Longitudinal study } \\
\text { 4. Cross-sectional study }\end{array}$ & $\begin{array}{l}\text { I. MtF GID adults before and after CSHT } \\
\text { 2. MtF GID adults with CSHT and after } \\
\text { withdrawal } \\
\text { 3. MtF GID adults before and after CSHT } \\
\text { 4. MtF GID adults with CSHT vs MtF } \\
\text { GID adults without CSHT }\end{array}$ & Mood states \\
\hline Newfield et $\mathrm{al}^{24}$ & Cross-sectional study & $\begin{array}{l}\text { FtM adults with CSHT vs FtM adults } \\
\text { without CSHT }\end{array}$ & Mental health-related quality of life \\
\hline Slabbekoorn et $\mathrm{al}^{31}$ & Longitudinal study & $\begin{array}{l}\text { MtF and FtM adults before and after } \\
\text { CSHT }\end{array}$ & $\begin{array}{l}\text { Emotional functioning, affect intensity, } \\
\text { anger readiness, nonverbal emotional } \\
\text { expressiveness, and mood states }\end{array}$ \\
\hline Blanchard et $\mathrm{al}^{34}$ & Cross-sectional study & MtF adults & $\begin{array}{l}\text { Psychological (depression and } \\
\text { tension) and social (cohabitation and } \\
\text { involvement) adjustment }\end{array}$ \\
\hline Leavitt et $\mathrm{al}^{30}$ & Cross-sectional study & $\begin{array}{l}\text { MtF adults with CSHT vs MtF adults } \\
\text { without CSHT }\end{array}$ & Personality-related psychopathology \\
\hline
\end{tabular}

Abbreviations: CSHT, cross-sex hormonal treatment; FtM, female-to-male; GD, gender dysphoria; GID, gender identity disorder; MtF, male-to-female. 


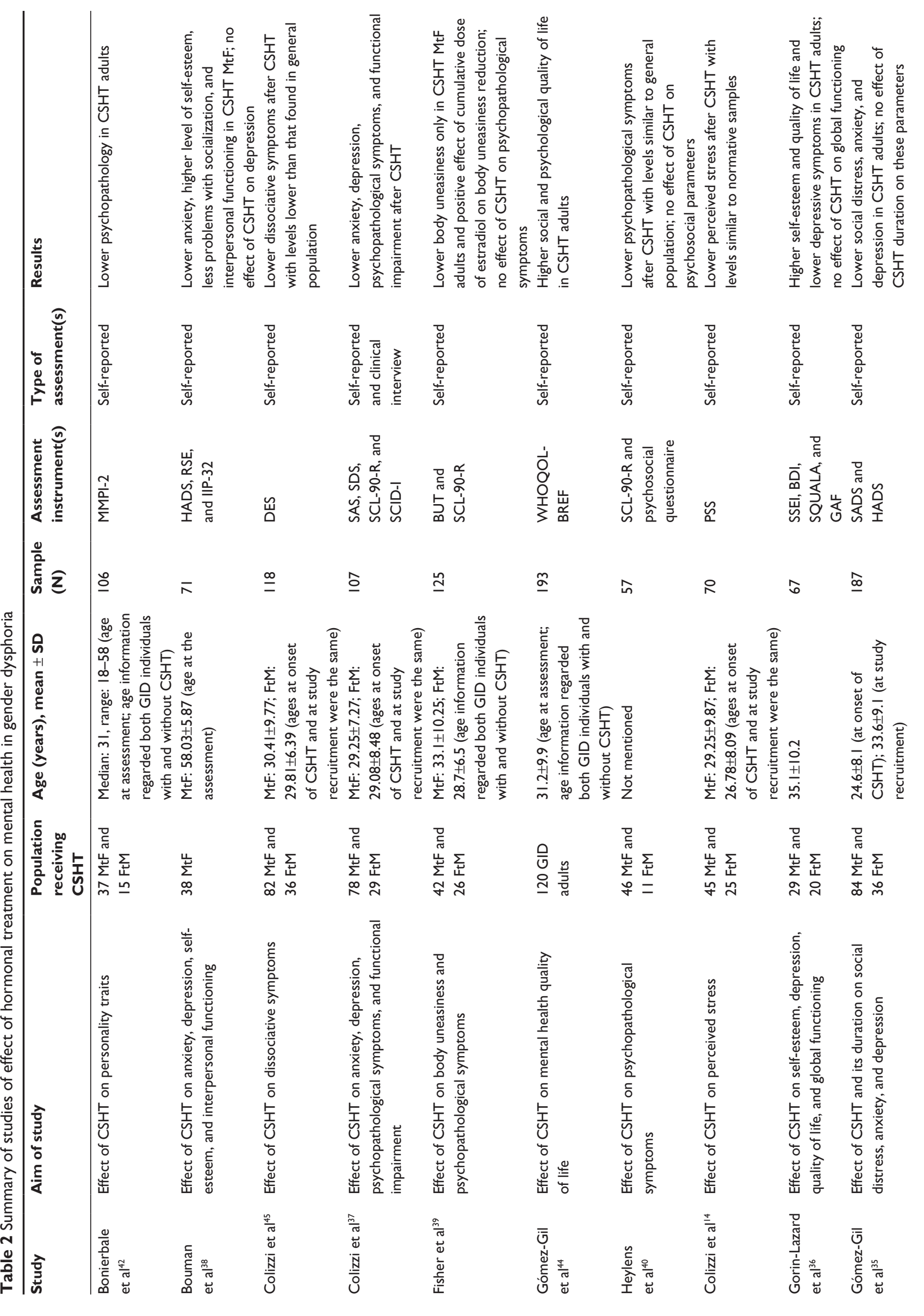




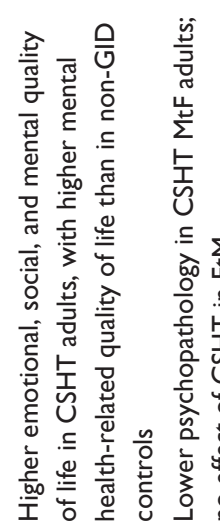

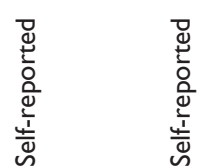

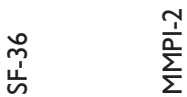

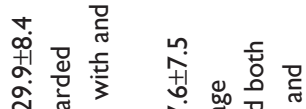

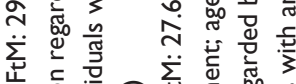

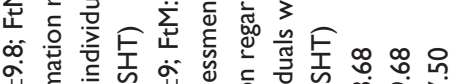

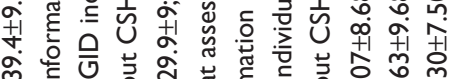

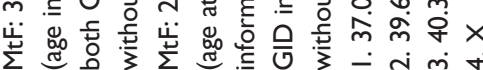

$\overline{0} \underline{\underline{0}} \quad \underline{9}$

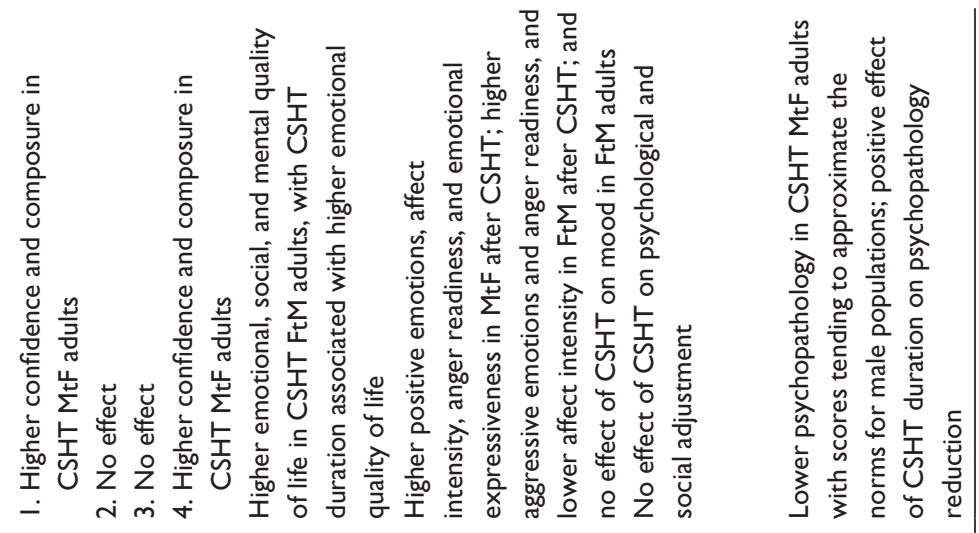

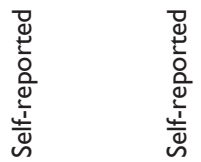

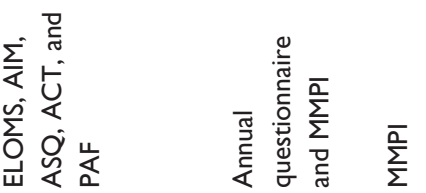

兽 $\overline{0}$ 另

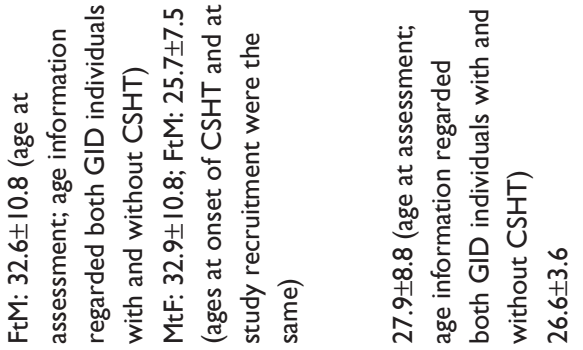

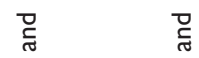

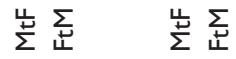

노은

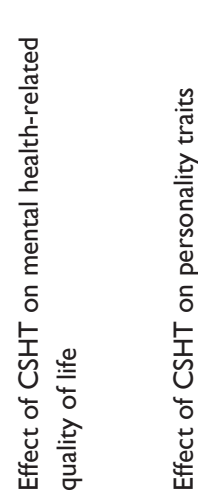

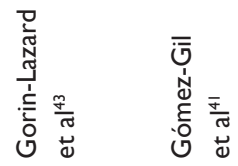

崖崖崖崖

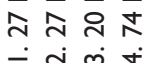

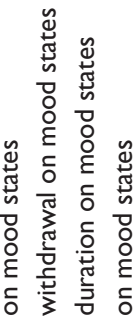

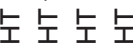

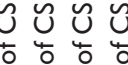

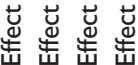

-

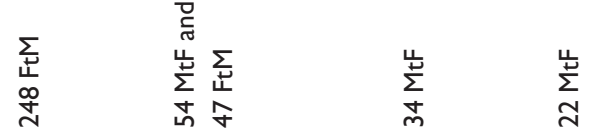

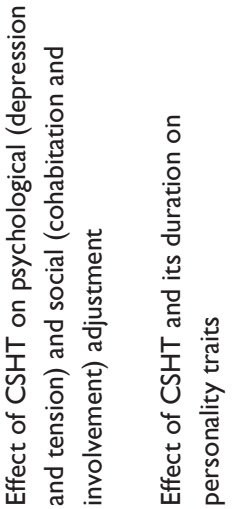

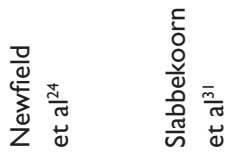

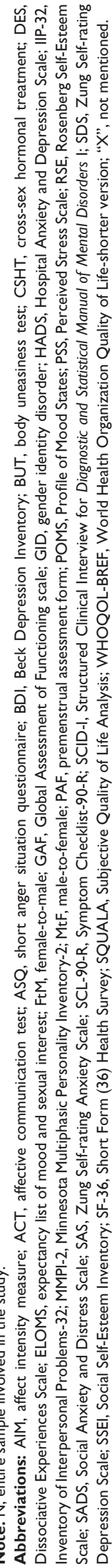


Table 3 Methodological quality of studies of effect of hormonal treatment on mental health in gender dysphoria

\begin{tabular}{|c|c|c|c|c|}
\hline Study & $\begin{array}{l}\text { Defined study } \\
\text { population }\end{array}$ & CSHT mean duration & CSHT type and dosage & Control \\
\hline $\begin{array}{l}\text { Bonierbale } \\
\text { et } \mathrm{al}^{42}\end{array}$ & $\begin{array}{l}\checkmark \text { GID formal } \\
\text { diagnosis at a gender } \\
\text { clinic according to } \\
\text { DSM-IV criteria }\end{array}$ & $\begin{array}{l}\checkmark / \times 3 \text { months at least } \\
\text { for both MtF and FtM }\end{array}$ & $\times$ Not mentioned & $\begin{array}{l}\checkmark / \times \text { No; comparison with } \\
16 \mathrm{MtF} \text { and } 38 \mathrm{FtM} \text { without } \\
\text { CSHT }\end{array}$ \\
\hline $\begin{array}{l}\text { Bouman } \\
\text { et } \mathrm{al}^{38}\end{array}$ & $\begin{array}{l}\checkmark / \times \text { Formal } \\
\text { diagnosis at a gender } \\
\text { clinic, criteria not } \\
\text { mentioned }\end{array}$ & $\times$ Not mentioned & $\begin{array}{l}\checkmark / \times M t F \text { : estrogens, tablet form or patches } \\
(n=2 I) \text {; sometimes in association with } \\
\text { cyproterone acetate, spironolactone, or } \\
\text { finasteride }(n=I I) \text {; and no information provided } \\
\text { for } I 7 \text { individuals on CSHT dosage, molecule } \\
\text { nature, or administration modalities }\end{array}$ & $\begin{array}{l}\checkmark / \times \text { No; comparison with } \\
33 \mathrm{MtF} \text { adults without CSHT }\end{array}$ \\
\hline Colizzi et al ${ }^{45}$ & $\begin{array}{l}\checkmark \text { GD formal } \\
\text { diagnosis at a gender } \\
\text { clinic according } \\
\text { to DSM-5 SCID-I } \\
\text { criteria }\end{array}$ & $\begin{array}{l}\checkmark 12 \text { months for both } \\
\text { MtF and FtM }\end{array}$ & $\begin{array}{l}\checkmark \mathrm{MtF}: \text { transdermal estradiol gel } \\
(\mathrm{I} .84 \pm 0.49 \mathrm{mg} / \mathrm{d}) \text { in association with oral } \\
\text { cyproterone acetate }(100 \mathrm{mg} / \mathrm{d}) \text { and FtM: IM } \\
\text { testosterone }(250 \mathrm{mg} \text { every } 26.3 \mathrm{I} \pm 2.68 \text { days })\end{array}$ & $\begin{array}{l}\checkmark / \times \text { No; normative } \\
\text { data from a sample of } \\
\text { I,055 subjects }\end{array}$ \\
\hline Colizzi et $\mathrm{al}^{37}$ & $\begin{array}{l}\checkmark \text { GID formal } \\
\text { diagnosis at a gender } \\
\text { clinic according } \\
\text { to DSM-IV SCID-I } \\
\text { criteria }\end{array}$ & $\begin{array}{l}\checkmark 12 \text { months for both } \\
\text { MtF and FtM }\end{array}$ & $\begin{array}{l}\checkmark \text { MtF: transdermal estradiol gel } \\
(I .82 \pm 0.53 \mathrm{mg} / \mathrm{d}) \text { in association with oral } \\
\text { cyproterone acetate }(100 \mathrm{mg} / \mathrm{d}) \text { and FtM: IM } \\
\text { testosterone }(250 \mathrm{mg} \text { every } 26.24 \pm 2.7 \mathrm{I} \text { days })\end{array}$ & $\times$ No \\
\hline Fisher et $\mathrm{al}^{39}$ & $\begin{array}{l}\checkmark \text { GD formal } \\
\text { diagnosis at different } \\
\text { gender clinics } \\
\text { according to DSM-IV } \\
\text { criteria }\end{array}$ & $\begin{array}{l}\checkmark \text { MtF: } 467 \pm 323 \text { days and } \\
\text { FtM: } 1,940 \pm 2,595 \text { days }\end{array}$ & $\begin{array}{l}\checkmark / \times \text { MtF: estradiol valerate }(n=\mid 2) \text {, transdermal } \\
\text { estradiol hemihydrate }(n=\mid 2) \text {, estradiol gel }(n=6) \text {, } \\
\text { and oral cyproterone acetate }(n=39) \text { and FtM: IM } \\
\text { testosterone enanthate }(n=I 2) \text {, IV testosterone } \\
\text { undecanoate }(n=I) \text {, and transdermal } \\
\text { testosterone }(n=9) \text {; no information on four FtM } \\
\text { and CSHT dosage }\end{array}$ & $\begin{array}{l}\checkmark / \times \text { No; comparison with } \\
57 \mathrm{GD} \text { adults without CSHT } \\
(24 \mathrm{MtF} \text { and } 33 \mathrm{FtM})\end{array}$ \\
\hline $\begin{array}{l}\text { Gómez-Gil } \\
\text { et } \mathrm{al}^{44}\end{array}$ & $\begin{array}{l}\checkmark \text { GID formal } \\
\text { diagnosis at a gender } \\
\text { clinic according to } \\
\text { DSM-IV and ICD-I0 } \\
\text { criteria }\end{array}$ & $\times$ Not mentioned & $\times$ Not mentioned & $\begin{array}{l}\checkmark / \times \text { No; comparison with } \\
73 \text { GID adults without CSHT }\end{array}$ \\
\hline $\begin{array}{l}\text { Heylens } \\
\text { et } \mathrm{al}^{40}\end{array}$ & $\begin{array}{l}\checkmark \text { GID formal } \\
\text { diagnosis at a gender } \\
\text { clinic according to } \\
\text { DSM-IV criteria }\end{array}$ & $\begin{array}{l}\checkmark / \times 3-6 \text { months for } \\
\text { both MtF and FtM }\end{array}$ & $\times$ Not mentioned & $\times$ No \\
\hline Colizzi et $\mathrm{al}^{14}$ & $\begin{array}{l}\checkmark \text { GID formal } \\
\text { diagnosis at a gender } \\
\text { clinic according } \\
\text { to DSM-IV SCID-I } \\
\text { criteria }\end{array}$ & $\begin{array}{l}\checkmark 12 \text { months for both } \\
\text { MtF and FtM }\end{array}$ & $\begin{array}{l}\checkmark \text { MtF: transdermal estradiol gel } \\
(1.77 \pm 0.46 \mathrm{mg} / \mathrm{d}) \text { in association with oral } \\
\text { cyproterone acetate }(100 \mathrm{mg} / \mathrm{d}) \text { and FtM: IM } \\
\text { testosterone }(250 \mathrm{mg} \text { every } 27.12 \pm 2.64 \text { days })\end{array}$ & $\begin{array}{l}\checkmark / \times \text { No; normative } \\
\text { data from a sample of } \\
645 \text { subjects }\end{array}$ \\
\hline $\begin{array}{l}\text { Gorin-Lazard } \\
\text { et } \mathrm{al}^{36}\end{array}$ & $\begin{array}{l}\checkmark \text { GID formal } \\
\text { diagnosis at a gender } \\
\text { clinic according to } \\
\text { DSM-IV criteria }\end{array}$ & $\begin{array}{l}\checkmark \mathrm{I} 2 \text { months at least for } \\
\text { both } \mathrm{MtF} \text { and } \mathrm{FtM}\end{array}$ & $\begin{array}{l}\checkmark / \times \text { MtF: cyproterone acetate followed } \\
\text { by estrogens combined with antiandrogens } \\
\text { (luteinizing hormone-releasing hormone analogs) } \\
\text { and FtM: synthetic progestagens followed by } \\
\text { testosterone (dosage, molecule nature, and } \\
\text { administration modalities of CSHT not reported) }\end{array}$ & $\begin{array}{l}\checkmark / \times \text { No; comparison with } \\
\text { I } 8 \text { GID adults without CSHT } \\
\text { (seven MtF and eleven FtM) }\end{array}$ \\
\hline
\end{tabular}




\begin{tabular}{l} 
Comparability of subjects \\
\hline$\checkmark$ Sociodemographic characteristics for \\
GID adults with and without CSHT not \\
reported, however, analyses controlled \\
for age, age on onset, and sexual \\
orientation \\
$\checkmark$ Significant difference in age of \\
referral, coming out, and transitioning; \\
sociodemographic characteristics \\
matched (ethnic group, employment, \\
civil status, and children)
\end{tabular}

$\checkmark$ Pre/postgroups were the same

$\checkmark$ Pre/postgroups were the same

$\begin{aligned} & \text { Statistical } \\ & \text { analysis }\end{aligned}$
$\checkmark$ Mann-Whitney
$t$-test, chi-square
or Fisher's exact
tests, and logistic
regression
$\checkmark$ Chi-square,
Mann-Whitney
$U$-test, and
MANCOVA

$\checkmark t$-test

$\begin{aligned} & \text { Mental health } \\ & \text { comorbidity }\end{aligned}$
$\times$ Not mentioned

$\checkmark$ Psychiatric history
was not exclusion
criterion, however, clinical
characteristics (psychiatric
history and self-harm)
matched
$\checkmark$ Unstable psychiatric

Attrition

$\checkmark / \times$ No available data in $26 \%$ of the original cohort $(\mathrm{N}=143)$ for different reasons $(\mathrm{N}=29$ patients not eligible for sex reassignment surgery; $\mathrm{N}=6$ with no valid MMPI; $\mathrm{N}=2$ missing data)

$\checkmark / \times$ No available data in $7.8 \%$ of the original cohort $(\mathrm{N}=77$; three did not attend appointment and three FtM excluded from analysis due to small number)

$\checkmark 0$ lost at recruitment or follow-up criterion

\author{
$\checkmark$ McNemar test \\ and $t$-test
}
$\checkmark$ Unstable psychiatric comorbidity was exclusion criterion

\section{$\checkmark$ ANCOVA}

$\checkmark$ Sociodemographic characteristics for GID adults with and without CSHT not reported, however, analyses controlled for gender, age, gender role, and surgery

$\checkmark$ Sociodemographic characteristics for GID adults with and without CSHT not reported, however, analyses controlled for gender, age, education, working/ student status, partnership status, and family support

$\checkmark / \times$ Pre/postgroups were not the same, no mention of possible differences between GID adults completing/not completing follow-up evaluation

\section{$\checkmark$ Pre/postgroups were the same $\quad \checkmark$ t-test

$$
\checkmark \text { t-test }
$$

$\checkmark$ Significant difference in age and sexual orientation, but corrected

\section{$\checkmark$ Multiple linear $\times$ Not mentioned regression}

$\checkmark$ Friedman test and Wilcoxon test $\times$ Not mentioned (mental retardation was exclusion criterion)

regression $\checkmark / \times 55 \%$ of original cohort $(\mathrm{N}=275)$ were excluded for different reasons (CSHT treatment prior to the study, disorder of sexual development, internalized homophobia, transvestite fetishism, mental retardation, dropout during the assessment, and completed genital reassignment surgery

$\checkmark / \times$ No available data in $30 \%$ of the original cohort $(\mathrm{N}=277)$ for different reasons $(\mathrm{N}=17$ patients refused to participate in the study; $\mathrm{N}=59$ were incomplete answers; and $\mathrm{N}=8$ previous genital surgery)

$\checkmark / \times$ No available data in $37 \%$ of the original cohort $(\mathrm{N}=90)$ for different reasons (refused to participate, attended the clinic once, GID NOS, and comorbidity) and $17.5 \%$ of the recruited subjects lost at follow-up

$\checkmark 0$ lost at recruitment or follow-up $\quad \checkmark$ Declared was exclusion criterion $\checkmark$ Psychiatric comorbidity $\checkmark$ Declared $\checkmark$ Declared $\checkmark$ Declared

$\checkmark$ Declared

$\checkmark$ Declared
Funding or

$\checkmark$ Declared

$\checkmark$ Declared

$\checkmark$ Declared

Declared

.

$\checkmark$ Mann-Whitney $\checkmark$ Psychotic disorder $U$-test and and unstable psychiatric multiple linear regression comorbidity (except nonmajor depressive disorder) were exclusion criteria

(Continued) 
Table 3 (Continued)

\begin{tabular}{|c|c|c|c|c|}
\hline Study & $\begin{array}{l}\text { Defined study } \\
\text { population }\end{array}$ & CSHT mean duration & CSHT type and dosage & Control \\
\hline $\begin{array}{l}\text { Gómez-Gil } \\
\text { et al }{ }^{35}\end{array}$ & $\begin{array}{l}\checkmark \text { GID formal } \\
\text { diagnosis at a gender } \\
\text { clinic according to } \\
\text { DSM-IV and ICD-I0 } \\
\text { criteria }\end{array}$ & $\begin{array}{l}\checkmark \text { MtF: II years and FtM: } \\
4.7 \text { years }\end{array}$ & $\begin{array}{l}\checkmark \text { MtF: oral estrogens (conjugated estrogens } \\
\text { I.8-2.4 mg/d or estradiol valerate } 2-4 \mathrm{mg} / \mathrm{d} \text { ) } \\
\text { or transdermal estradiol patches }(3 \mathrm{mg} \text { twice } \\
\text { per week, delivering } 100 \mathrm{mg} / \mathrm{d} \text { ), generally in } \\
\text { association with oral cyproterone acetate } \\
(25-50 \mathrm{mg} / \mathrm{d}) \text { and FtM: IM testosterone } \\
(1,000 \mathrm{mg} \text { every } 10-14 \text { weeks), or daily } \\
\text { transdermal testosterone gel }(50 \mathrm{mg} / \mathrm{d})\end{array}$ & $\begin{array}{l}\checkmark / \times \text { No; comparison with } \\
67 \mathrm{GID} \text { adults without CSHT } \\
(29 \mathrm{MtF} \text { and } 38 \mathrm{FtM})\end{array}$ \\
\hline $\begin{array}{l}\text { Gorin-Lazard } \\
\text { et al }{ }^{43}\end{array}$ & $\begin{array}{l}\checkmark \text { GID formal } \\
\text { diagnosis at a gender } \\
\text { clinic according to } \\
\text { DSM-IV MINI criteria }\end{array}$ & $\begin{array}{l}\checkmark \mathrm{I} 2 \text { months at least for } \\
\text { both MtF and FtM }\end{array}$ & $\begin{array}{l}\checkmark / \times \text { MtF: antiandrogens along with estrogens } \\
\text { and FtM: synthetic progestagens with } \\
\text { testosterone (dosage, molecule nature, and } \\
\text { administration modalities of CSHT not reported) }\end{array}$ & $\begin{array}{l}\checkmark / \times \text { No; comparison with } \\
\text { I7 GID adults without CSHT } \\
\text { (six MtF and eleven FtM); } \\
\text { controls from a normative } \\
\text { sample of } 3,656 \text { subjects }\end{array}$ \\
\hline $\begin{array}{l}\text { Gómez-Gil } \\
\text { et } \mathrm{al}^{41}\end{array}$ & $\begin{array}{l}\checkmark \text { GID formal } \\
\text { diagnosis at a gender } \\
\text { clinic according to } \\
\text { DSM-IV criteria }\end{array}$ & $\begin{array}{l}\checkmark 12 \text { months at least for } \\
\text { both MtF and FtM }\end{array}$ & $\times$ Not mentioned & $\begin{array}{l}\checkmark / \times \text { No; comparison with } \\
84 \mathrm{GID} \text { adults without CSHT } \\
(38 \mathrm{MtF} \text { and } 46 \mathrm{FtM})\end{array}$ \\
\hline Miles et a ${ }^{12}$ & $\begin{array}{l}\checkmark \text { GID formal } \\
\text { diagnosis at a gender } \\
\text { clinic according to } \\
\text { DSM-IV criteria }\end{array}$ & $\begin{array}{l}\text { I. } \checkmark / \times 3-12 \text { months for } \\
\text { both MtF and FtM } \\
\text { 2. } \checkmark / \times \text { at least } 8 \text { weeks } \\
\text { of withdrawal after at } \\
\text { least } 28 \text { months of CSHT } \\
\text { for both MtF and FtM } \\
\text { 3. } \checkmark / \times 6-I 5 \text { months for } \\
\text { both MtF and FtM } \\
\text { 4. } \times\end{array}$ & $\begin{array}{l}\checkmark \text { Conjugated equine estrogens }(1.25-7.5 \mathrm{mg} / \mathrm{d}) \\
\text { or ethinyl estradiol }(10-15 \mu \mathrm{g} / \mathrm{d}) \text {, sometimes } \\
\text { in association with cyproterone acetate } \\
(50-150 \mathrm{mg} / \mathrm{d}) \text { or medroxyprogesterone } \\
\text { acetate }(15 \mathrm{mg} / \mathrm{d})\end{array}$ & $\begin{array}{l}\checkmark / \times \text { No } \\
\text { 4. Comparison with MtF } \\
\text { without CSHT }\end{array}$ \\
\hline $\begin{array}{l}\text { Newfield } \\
\text { et a }\left.\right|^{24}\end{array}$ & $\times$ Not mentioned & $\begin{array}{l}\checkmark / X<5 \text { years for the } \\
\text { majority of FtM }(n=203)\end{array}$ & $\times$ Not mentioned & $\begin{array}{l}\checkmark / \times \text { No; comparison with } \\
\text { I I FtM adults without CSHT }\end{array}$ \\
\hline $\begin{array}{l}\text { Slabbekoorn } \\
\text { et al }{ }^{31}\end{array}$ & $\begin{array}{l}\checkmark / \times \text { Formal } \\
\text { diagnosis at a gender } \\
\text { clinic, criteria not } \\
\text { mentioned }\end{array}$ & $\begin{array}{l}\checkmark / \times I 4 \text { weeks for both } \\
M t F \text { and FtM }\end{array}$ & $\begin{array}{l}\checkmark \text { MtF: oral cyproterone acetate }(50 \mathrm{mg} / \text { twice } \\
\text { a day) in combination with oral ethinyl estradiol } \\
(0.05 \mathrm{mg} / \text { twice a day, } \mathrm{n}=32) \text { or } 17 \beta \text {-estradiol } \\
\text { plasters }(0.1 \mathrm{mg} / \mathrm{d}, \mathrm{n}=22) ; \mathrm{FtM} \text { : IM testosterone } \\
\text { esters }(250 \mathrm{mg} / 2 \text { weeks, } \mathrm{n}=42), \text { or oral } \\
\text { undecanoate testosterone }(200 \mathrm{mg} / \mathrm{d}, \mathrm{n}=5)\end{array}$ & $\times$ No \\
\hline $\begin{array}{l}\text { Blanchard } \\
\text { et } \mathrm{al}^{34}\end{array}$ & $\begin{array}{l}\checkmark / \times \text { Formal } \\
\text { diagnosis at a gender } \\
\text { clinic, criteria not } \\
\text { mentioned }\end{array}$ & $\times$ Not mentioned & $\times$ Not mentioned & $\begin{array}{l}\checkmark / \times \mathrm{No} \text {; comparison with } \\
2 \mathrm{I} \mathrm{MtF} \text { adults without } \mathrm{CSHT}\end{array}$ \\
\hline Leavitt et $\mathrm{a}^{30}$ & $\times$ Not mentioned & $\checkmark 12$ months at least & $\begin{array}{l}\checkmark / \times \text { Oral conjugated estrogens and } \\
\text { medroxyprogesterone (cyclically each month in a } \\
\text { dose sufficient to inhibit spontaneous erections) }\end{array}$ & $\begin{array}{l}\checkmark / \times \mathrm{No} \text {; comparison with } \\
19 \mathrm{MtF} \text { adults without } \mathrm{CSHT}\end{array}$ \\
\hline
\end{tabular}

Note: $\checkmark$, good quality; $\checkmark / \times$, fair quality; $\times$, poor quality.

Abbreviations: ANCOVA, analysis of covariance; ANOVA, analysis of variance; CSHT, cross-sex hormonal treatment; DSM, Diagnostic and Statistical Manual of Mental Disorders; FtM, female to male; GD, gender dysphoria; GID, gender identity disorder; ICD-I0, international classification of diseases - I0th revision; IM, intramuscular; IV, intravenous; MANCOVA, multivariate analysis of covariance; MINI, mini-international neuropsychiatric interview; MMPI-2, Minnesota Multiphasic Personality Inventory-2; MtF, male to female; NOS, not otherwise specified; SCID-I, Structured Clinical Interview for DSM I. 


\begin{tabular}{|c|c|c|c|c|}
\hline Comparability of subjects & $\begin{array}{l}\text { Statistical } \\
\text { analysis }\end{array}$ & $\begin{array}{l}\text { Mental health } \\
\text { comorbidity }\end{array}$ & Attrition & $\begin{array}{l}\text { Funding or } \\
\text { sponsorship }\end{array}$ \\
\hline $\begin{array}{l}\checkmark \text { Significant difference in age, MtF } \\
\text { prevalence, and level of education } \\
\text { (without CSHT < CSHT), but } \\
\text { these were corrected. Other } \\
\text { sociodemographic characteristics } \\
\text { matched (living arrangements, sexual } \\
\text { orientation, and employment status) }\end{array}$ & $\begin{array}{l}\checkmark \text { ANOVA/ } \\
\text { ANCOVA, } \\
\text { chi-square, } \\
\text { and Pearson's } \\
\text { correlation }\end{array}$ & $\times$ Not mentioned & $\begin{array}{l}\checkmark / \times \text { No available data in } 6.5 \% \text { of } \\
\text { original cohort }(\mathrm{N}=200)\end{array}$ & $\checkmark$ Declared \\
\hline $\begin{array}{l}\checkmark \text { Sociodemographic characteristics } \\
\text { matched (age for GID adults with and } \\
\text { without CSHT and age and sex for GID } \\
\text { adults and non-GID controls) }\end{array}$ & $\begin{array}{l}\checkmark \text { Mann-Whitney } \\
\text { test, multiple } \\
\text { linear regression, } \\
\text { and } t \text {-test }\end{array}$ & $\begin{array}{l}\checkmark \text { Psychotic disorder } \\
\text { and unstable psychiatric } \\
\text { comorbidity (except } \\
\text { non major depressive } \\
\text { disorder) were exclusion } \\
\text { criteria }\end{array}$ & $\begin{array}{l}\checkmark / \times \text { No available data in } 9 \% \text { of original } \\
\text { cohort }(N=67)\end{array}$ & $\checkmark$ Declared \\
\hline$\times$ Not mentioned & $\checkmark$ t-test & $\times$ Not mentioned & $\begin{array}{l}\checkmark / \times \text { No available data in } 36 \% \text { of the } \\
\text { original cohort }(\mathrm{N}=254) \text { for different } \\
\text { reasons }(\mathrm{N}=24 \text { patients failed to meet } \\
\text { GID DSM-IV criteria; } \mathrm{N}=24 \text { patients } \\
\text { were administered the MMPI instead of } \\
\text { MMPI-2; and } \mathrm{N}=43 \text { were missing data) }\end{array}$ & $\begin{array}{l}\times \text { Not } \\
\text { declared }\end{array}$ \\
\hline $\begin{array}{l}\text { I., 2., and 3. } \checkmark \text { Pre/postgroups were } \\
\text { the same } \\
\text { 4. } \times \text { Significant difference in age, not } \\
\text { corrected }\end{array}$ & $\begin{array}{l}\checkmark \text { MANOVAs } \\
\text { and Pearson's } \\
\text { correlation }\end{array}$ & $\times$ Not mentioned & $\checkmark 0$ lost at recruitment or follow-up & $\checkmark$ Declared \\
\hline $\begin{array}{l}\checkmark / \times \text { Sociodemographic characteristics } \\
\text { not reported; analyses were controlled } \\
\text { for income and education }\end{array}$ & $\begin{array}{l}\checkmark \text { ANOVA/ } \\
\text { ANCOVA }\end{array}$ & $\times$ Not mentioned & $\begin{array}{l}\checkmark / \times \text { No available data in I } 8 \% \text { of the } \\
\text { original cohort }(\mathrm{N}=446) \text { for different } \\
\text { reasons (not from the US, identified } \\
\text { as female/no gender information, no } \\
\text { information on quality of life or } \mathrm{CSHT} \text { ) }\end{array}$ & $\begin{array}{l}\times \text { Not } \\
\text { declared }\end{array}$ \\
\hline$\checkmark$ Pre/postgroups were the same & $\begin{array}{l}\checkmark \text { ANOVA and } \\
t \text {-test }\end{array}$ & $\times$ Not mentioned & $\checkmark 0$ lost at recruitment or follow-up & $\begin{array}{l}\times \text { Not } \\
\text { declared }\end{array}$ \\
\hline $\begin{array}{l}\checkmark \text { Sociodemographic characteristics for } \\
\text { GID adults with and without CSHT not } \\
\text { reported, however, analyses controlled } \\
\text { for age and social feminization }\end{array}$ & $\begin{array}{l}\checkmark \text { Multiple } \\
\text { regression }\end{array}$ & $\begin{array}{l}\checkmark / \times \text { Not mentioned } \\
\text { (gender complaint-related } \\
\text { psychotic delusion was } \\
\text { exclusion criterion) }\end{array}$ & $\begin{array}{l}\checkmark / \times \text { No available data in } 44 \% \text { of the } \\
\text { original cohort }(\mathrm{N}=98) \text { for different } \\
\text { reasons ( } \mathrm{N}=42 \text { patients excluded } \\
\text { because of not expressing - clear - } \\
\text { homosexual orientation; } \mathrm{N}=\mathrm{I} \text { excluded } \\
\text { due to Klinefelter's syndrome) }\end{array}$ & $\begin{array}{l}\times \text { Not } \\
\text { declared }\end{array}$ \\
\hline $\begin{array}{l}\checkmark \text { Sociodemographic characteristics } \\
\text { matched (age and education) }\end{array}$ & $\begin{array}{l}\checkmark \text { t-test and } \\
\text { Pearson's } \\
\text { correlation }\end{array}$ & $\times$ Not mentioned & $\checkmark 0$ lost at recruitment & $\begin{array}{l}\times \text { Not } \\
\text { declared }\end{array}$ \\
\hline
\end{tabular}


and body uneasiness. Key findings are reported in Table 2, and a brief synopsis of them is reported later.

\section{Effect of cross-sex hormonal treatment on depression, mood states, and self-esteem}

Depression and its features have been the most extensively investigated mental health-related parameters in gender dysphoria individuals receiving hormonal treatment. The first study to investigate this issue was carried out in 1983. ${ }^{34}$ This cross-sectional study assessed male-to-female (MtF) individuals' psychological adjustment depending on their hormonal treatment status. The research did not find any significant difference in terms of depressive symptoms between MtFs with and without hormonal treatment. ${ }^{34} \mathrm{In}$ a second study, Slabbekoorn et $\mathrm{al}^{31}$ asked female-to-male (FtM) individuals to keep a mood diary during their testosterone administration. Results indicated no mood changes when assessing depressed mood and other mood-related parameters from baseline to after the fourth testosterone injection. ${ }^{31}$ Miles et a ${ }^{29}$ used a unique design to disentangle the hormonal treatment contribution to mood in gender dysphoria. Using both independent groups (longitudinal study) and repeated measure (cross-sectional study) designs, this study consistently indicated that hormone-treated MtFs are more composed and confident. Authors also tested for the effect of hormonal treatment withdrawal and duration on mood. Intriguingly, they reported no significant association. ${ }^{29}$ In recent years, four more studies have investigated the association between hormonal treatment and depression, using commonly used questionnaires/scales. ${ }^{35-38}$ Apart from a study, ${ }^{38}$ which reported no significant differences in terms of depression (it only investigated MtFs), the remaining studies reported lower depressive symptoms in gender dysphoria individuals receiving hormonal treatment. ${ }^{35-37}$ Two of these studies also assessed self-esteem, reporting higher levels of self-esteem due to the hormonal treatment intervention. ${ }^{36,38}$

\section{Effect of cross-sex hormonal treatment on anxiety and tension}

Different from depression, anxiety has been investigated only recently, with three studies addressing this aspect over the last 5 years. ${ }^{35,37,38}$ All these three investigations (two cross-sectional studies and one longitudinal one) have consistently reported a reduction in symptoms of anxiety among individuals receiving hormone therapy. A previous research had studied tension as a measure of psychological adjustment, reporting no significant differences between MtFs with and without hormonal treatment. ${ }^{34}$

\section{Effect of cross-sex hormonal treatment on global and personality-related psychopathology}

This review has identified three recent studies about the effect of cross-sex hormonal treatment on a global measure of psychopathology. ${ }^{37,39,40}$ Intriguingly, all these studies used the same questionnaire. However, while two studies described a significant decrease in global psychopathology occurring after the initiation of hormone therapy, ${ }^{37,40}$ a third one did not find any difference between hormone-treated and nonhormone-treated individuals. ${ }^{39}$ Interestingly, according to the authors, their negative finding may be attributable to the restricted range and low levels of psychopathology observed in their sample.

Three other studies investigated personality-related psychopathology as a function of cross-sex hormonal treatment, using a similar methodology. ${ }^{30,41,42}$ Using a common measure of personality, this body of research has suggested a reduction in personality-related psychopathology among gender dysphoria individuals receiving hormone therapy. However, as one study focused only on MtF individuals ${ }^{30}$ and a second study reported hormonal treatment to be beneficial only in $\mathrm{MtF}$ and not in FtM individuals, ${ }^{41}$ there is still little evidence to suggest a positive effect of cross-sex hormonal treatment on FtM individuals' personality-related psychopathology. ${ }^{42}$ Leavitt et $\mathrm{al}^{30}$ also indicated that the hormone-treated MtF individuals' overall personality-related psychopathology tends to approximate the norms for male populations and negatively correlates with the hormone therapy duration.

\section{Effect of cross-sex hormonal treatment on mental health-related quality of life}

The first study to investigate the quality of life among gender dysphoria individuals receiving hormonal treatment was performed in 2006 on a large sample of FtM individuals. ${ }^{24}$ With $\sim 250$ FtMs receiving cross-sex hormonal treatment, this study sample represents the largest one identified in this systematic review. The research reported higher emotional, social, and mental quality of life in hormone-treated FtM adults, with hormonal treatment duration associated with higher emotional quality of life. ${ }^{24}$ Three more recent studies confirmed a better psychosocial and mental quality of life in both MtFs and FtMs receiving hormone therapy. ${ }^{36,43,44}$ One study also compared hormone-treated individuals with 
a control group, reporting a higher mental health-related quality of life in gender dysphoria individuals. ${ }^{43}$

\section{Effect of cross-sex hormonal treatment on dissociative symptoms}

To date, only one study has investigated the prevalence of dissociative symptoms among gender dysphoria individuals receiving cross-sex hormonal treatment. ${ }^{45}$ Results of this longitudinal study suggest that suffering from dissociative symptoms may be a common clinical feature in the context of untreated gender dysphoria. Interestingly, this research indicated a significant reduction in gender dysphoria individuals' dissociative symptoms after the beginning of cross-sex hormonal treatment. It is worth reporting that the high prevalence of dissociative symptoms among untreated gender dysphoria individuals was greatly due to the genderrelated sense of strangeness. This issue could explain why the dissociative experience was less intense when gender dysphoria individuals received hormone therapy. The unease with the biological sex and the aversion to the corresponding sex-specific body forms belong by definition to the gender dysphoria diagnosis. ${ }^{2}$ Therefore, authors argued whether dissociation represents a genuine feature of gender dysphoria rather than a clinically relevant pathological manifestation, highlighting the need for additional/exclusion criteria for a differential diagnosis. ${ }^{45}$

\section{Effect of cross-sex hormonal treatment on psychosocial, interpersonal, and emotional functioning}

Psychosocial functioning has been the domain receiving most attention after depression in hormone-treated individuals. Different aspects have been investigated, such as emotional ${ }^{31}$ and social components. ${ }^{34,38}$ Also two studies have investigated a global measure of psychosocial functioning. ${ }^{36,37}$ Impact of hormone therapy on emotional functioning appeared to be different in $\mathrm{MtF}$ and FtM individuals, with more generally positive effects in MtF and some undesirable effects in FtM individuals. ${ }^{31}$ While Blanchard et $\mathrm{al}^{34}$ did not find any effect of hormone therapy on social adjustment, a more recent study has identified less problems with socialization and interpersonal functioning in gender dysphoria individuals receiving hormone therapy. ${ }^{38}$ Finally, mixed results have emerged from studies investigating global psychosocial functioning, with a study reporting less functional impairment after the beginning of hormone therapy ${ }^{37}$ and another one finding no significant differences as a result of hormonal treatment. ${ }^{36}$

\section{Effect of cross-sex hormonal treatment on social distress, perceived stress, and body uneasiness}

This review identified three studies specifically analyzing distress intensity among gender dysphoria individuals and how it could be influenced by the hormonal treatment intervention. ${ }^{14,35,39}$ These studies used the following three different measures of distress: 1) social distress, ie, avoidance and subjective distress about social interactions; 2) perceived stress, ie, perception of social life experiences as stressful and unpredictable/uncontrollable; and 3) body uneasiness, ie, gender-related distress and discomfort. Using a crosssectional design, Gómez-Gil et a ${ }^{35}$ reported that gender dysphoria individuals who have not received hormonal treatment show higher levels of social distress than those receiving cross-sex hormones. Consistent with these findings, ${ }^{35}$ in a second study, hormone-treated gender dysphoria individuals showed lower perceived stress, which overlapped with that found in the normative sample. ${ }^{14}$ The last study addressed the impact of hormonal treatment on body-related distress, suggesting a gender-specific effect. In fact, $\mathrm{MtF}$ individuals' body uneasiness was lower in the context of cross-sex hormonal treatment, and the reduction correlated negatively with the cumulative dose of estradiol. ${ }^{39}$ Instead, FtM subjects did not appear to benefit from the hormone therapy in terms of body uneasiness. Authors themselves commented on it as an unexpected finding, which could be due to the individual's still unsatisfactory comfort with the body at a private/personal level rather than involving a social dimension. ${ }^{39}$

\section{Risk of systematic bias across studies}

While generally well designed, results mentioned earlier need to be considered in light of certain limitations. Apart from one study, ${ }^{39}$ all the others came from only one clinic and some of them had a relatively small sample size. Most importantly, as no study used a blinded randomized controlled trial design, results could have also different explanations because of the study design(s). Moreover, type and dosage of crosssex hormonal treatment were often not reported, and when reported, there was poor consistency across studies. The same weakness applied to hormone therapy duration. While differences between analyzed groups were generally taken into account, more than half the studies did not mention/control for psychiatric comorbidity, which could have represented a critical bias in this kind of investigation. Finally, while studies were generally strict on the defined study population (formal diagnosis according to DSM criteria used at the time 
of the study), recruitment/follow-up attrition represented an issue for some of the studies identified in this review.

\section{Discussion}

This is the first systematic review of all studies examining the effect of cross-sex hormonal treatment on mental health and psychosocial well-being in gender dysphoria. A previous review has mainly focused on the psychosocial outcome of gender dysphoria individuals receiving sex reassignment surgery. ${ }^{27}$ After the publication of that review, the literature has seen an increasing number of studies specifically interested in whether sex hormones induce any improvement in terms of mental health and psychosocial well-being. Overall, this review demonstrates that cross-sex hormonal treatment has definite effects on mental health, generally ameliorating gender dysphoria individuals' well-being at different levels. Taking into account the risks of bias reported earlier, crosssex hormonal treatment does not seem to affect any of the parameters investigated, except emotional functioning, with a reported higher prevalence of aggressive emotions and lower affect intensity in FtMs receiving hormonal treatment. ${ }^{31}$

More specifically, when treated with hormone therapy, gender dysphoria individuals reported less anxiety, ${ }^{35,37,38}$ dissociation, ${ }^{45}$ perceived stress, ${ }^{14}$ social distress, ${ }^{35}$ and higher mental health-related quality of life $\mathrm{e}^{24,36,43,44}$ and self-esteem. ${ }^{36,38}$ Also, compared to FtM individuals, MtF individuals seemed to benefit more in terms of body uneasiness, ${ }^{39}$ emotional functioning, ${ }^{31}$ and personality-related psychopathology. ${ }^{30,41,42}$ More mixed results emerged from studies investigating other mental health-related dimensions. In particular, recent studies suggested reduced depressive symptoms in hormone-treated gender dysphoria individuals, ${ }^{35-37}$ despite previous evidence of no association between cross-sex hormonal treatment and depression among $\mathrm{MtF}^{34}$ and $\mathrm{FtM}$ individuals. ${ }^{31}$ Similarly, global psychopathology appeared to be reduced in two studies, ${ }^{8,37}$ while a third one did not detect any change. ${ }^{39}$ Finally, while two studies indicated less functional impairment ${ }^{37}$ and reduced problems with socialization and interpersonal functioning in gender dysphoria individuals receiving hormone therapy, ${ }^{38}$ two other studies did not find any effect of hormone therapy on social adjustment ${ }^{34}$ and psychosocial functioning. ${ }^{36}$ Negative findings generally came from studies involving a crosssectional design. On the contrary, the few longitudinal studies identified in this review were consistent in indicating an association between hormonal treatment and better mental health. ${ }^{14,29,31,37,40,45}$ Conducting observations of the same subjects over a period of time, longitudinal studies can establish sequences of events and better detect changes in the characteristics of the target population, also due to their higher statistical power.

Some of the studies included in this review offered a comparison of hormone-treated gender dysphoria individuals to normal range values of normative data for a general population. Interestingly, all these studies indicated that after the beginning of the cross-sex hormonal treatment, gender dysphoria individuals express distress levels similar to the general population in several mental health-related dimensions, including perceived stress, ${ }^{14}$ dissociative symptoms, ${ }^{45}$ personality-related psychopathology, ${ }^{30}$ mental health-related quality of life, ${ }^{43}$ and global psychopathology. ${ }^{40}$ These results are in-line with the majority of the previous studies, indicating that hormone therapy decreases the psychiatric comorbidities often associated with a lack of hormonal treatment. ${ }^{27}$

The finding of a reduced mental distress in the context of hormone-treated gender dysphoria may have several explanations. The question arising is whether cross-sex hormones exert a direct effect on distress levels, implying a biological explanation for their reduction, or rather indirectly elicit a better mental health, implying a psychological mechanism possibly related to the physical changes. Biologically, sex hormones may exert different effects on mental distress. Specifically, estrogens may increase mental distress, making individuals more prone to anxiety and depression. ${ }^{46}$ Instead, androgens can reduce mental distress, promoting feelings of euphoria and energy ${ }^{47}$ and also eliciting stress and hostility. ${ }^{48}$ As a consequence, mental distress should increase in $\mathrm{MtF}$ individuals as a consequence of the biological effect of estrogens. Conversely, due to the biological effects of androgens, hormone therapy should induce both positive and negative effects on FtM individuals' mental distress, depending on the mental health parameters evaluated. The only finding in support of a biological mechanism for the observed changes in mental distress is the increase in the prevalence of aggressive emotions among hormonetreated FtM individuals. ${ }^{31}$ Nevertheless, studies included in this review found that hormonal treatment has a positive effect on both MtF and FtM individuals' mental health. In addition, this amelioration is more pronounced in $\mathrm{MtF}$ individuals, who should be more negatively affected by the cross-sex hormonal treatment intervention according to the biological explanation. In light of all this evidence, this review does not support the hypothesis of a direct effect of hormone therapy on mental well-being in gender dysphoria. Instead, the distress reduction might be an indirect effect of hormone therapy, as already hypothesized by Kuiper 
and Cohen-Kettenis. ${ }^{32}$ Mental distress may be considered a reaction to the nonsatisfaction arising by the incongruence between the individual's experienced gender and biological sex. Cross-sex hormonal treatment is supposed to mitigate such incongruence, inducing desired physical changes that could eventually be responsible for the individual's better mental state and psychological well-being. In other words, thanks to the body changes obtained, gender dysphoria individuals may experience a reduction in their mental distress. In addition, other psychological explanations support the hypothesis of an indirect effect of hormonal treatment on mental well-being. Due to incongruence between their physical appearance and internal experience, nonhormone-treated gender dysphoria individuals may face major difficulties in managing their gender identity at a social level. Hormonal treatment may reinforce the gender affirmation with a better social recognition. This would explain why in some studies hormonal treatment seems to have a beneficial effect on the social dimension rather than on the private dimension of mental well-being. ${ }^{39}$ Finally, as delaying hormonal treatment has been reported to often disappoint gender dysphoria individuals, also increasing their distress, ${ }^{37,45}$ we cannot exclude that accessing care and receiving eligibility for hormone therapy may help in reducing distress, representing an acceptance of the individual's needs and requirements. In order to promote the highest standards of health for individuals seeking gender transitioning, the importance of facilitating access to care and reducing barriers across countries and cultures cannot be overemphasized. Several factors have been identified, which can affect gender dysphoria individuals' access to care and eventually lead to a nonsatisfactory outcome, such as stigma, violence, fear of being seen as different, lack of/ inadequate access to specialized health care professionals and/or services, and poor access to health care pathwayrelated information. ${ }^{49}$

\section{Conclusion}

This review suggests that gender dysphoria-related mental distress may benefit from hormonal treatment intervention. Even recognizing the suffering of nonhormone-treated gender dysphoria individuals, which represents a possible risk factor for more severe or full-syndromal psychiatric disorders, ${ }^{12}$ mental distress in gender dysphoria may be considered as a transient reaction to the nonsatisfaction connected to the incongruent body image and not necessarily a stable psychiatric comorbidity. In this perspective, timely hormonal treatment intervention represents a crucial issue in gender dysphoria individuals' mental health-related outcome.

\section{Acknowledgments}

The authors would like to thank all patients and colleagues met during the years of clinical research activity. A special thank goes to the Gender Unit at the University of Bari, Bari, Italy, and the Gender Identity Development Service at the Tavistock and Portman NHS Foundation Trust, London, UK. There was no funding source for this study.

\section{Disclosure}

The authors report no conflicts of interest in this work.

\section{References}

1. American Psychiatric Association (APA). Diagnostic and Statistical Manual of Mental Disorders: DSM-III. 3rd ed. Washington, DC: American Psychiatric Association; 1980.

2. American Psychiatric Association (APA). Diagnostic and Statistical Manual of Mental Disorders: DSM-5. 5th ed. Arlington, VA: American Psychiatric Association; 2013.

3. American Psychiatric Association (APA). Diagnostic and Statistical Manual of Mental Disorders: DSM-IV. 4th ed. Washington, DC: American Psychiatric Association; 1994.

4. American Psychiatric Association (APA). Diagnostic and Statistical Manual of Mental Disorders: DSM-IV-TR. 4th ed. Washington, DC: American Psychiatric Association; 2000.

5. Drescher J. Queer diagnoses: parallels and contrasts in the history of homosexuality, gender variance, and the diagnostic and statistical manual. Arch Sex Behav. 2010;39(2):427-460.

6. à Campo J, Nijman H, Merckelbach H, Evers C. Psychiatric comorbidity of gender identity disorders: a survey among Dutch psychiatrists. $\mathrm{Am}$ J Psychiatry. 2003;160(7):1332-1336.

7. Hepp U, Kraemer B, Schnyder U, Miller N, Delsignore A. Psychiatric comorbidity in gender identity disorder. J Psychosom Res. 2005;58(3):259-261.

8. Heylens G, Elaut E, Kreukels BP, et al. Psychiatric characteristics in transsexual individuals: multicentre study in four European countries. Br J Psychiatry. 2014;204(2):151-156.

9. Gómez-Gil E, Trilla A, Salamero M, Godás T, Valdés M. Sociodemographic, clinical, and psychiatric characteristics of transsexuals from Spain. Arch Sex Behav. 2009;38(3):378-392.

10. Hoshiai M, Matsumoto Y, Sato T, et al. Psychiatric comorbidity among patients with gender identity disorder. Psychiatry Clin Neurosci. 2010; 64(5):514-519.

11. Fisher AD, Bandini E, Casale H, et al. Sociodemographic and clinical features of gender identity disorder: an Italian multicentric evaluation. J Sex Med. 2013;10(2):408-419.

12. Dhejne C, Van Vlerken R, Heylens G, Arcelus J. Mental health and gender dysphoria: a review of the literature. Int Rev Psychiatry. 2016;28(1): 44-57.

13. Sohn M, Bosinski HA. Gender identity disorders: diagnostic and surgical aspects. J Sex Med. 2007;4(5):1193-1207.

14. Colizzi M, Costa R, Pace V, Todarello O. Hormonal treatment reduces psychobiological distress in gender identity disorder, independently of the attachment style. $J$ Sex Med. 2013;10(12):3049-3058.

15. Byne W, Bradley SJ, Coleman E, et al. Report of the American Psychiatric Association task force on treatment of gender identity disorder. Arch Sex Behav. 2012;41(4):759-796.

16. Cohen-Kettenis PT, van Goozen SH. Sex reassignment of adolescent transsexuals: a follow-up study. $J$ Am Acad Child Adolesc Psychiatry. 1997;36(2):262-271.

17. Rehman J, Lazer S, Benet AE, Schaefer LC, Melman A. The reported sex and surgery satisfactions of 28 postoperative male-to-female transsexual patients. Arch Sex Behav. 1999;28(1):71-89. 
18. Lawrence AA. Factors associated with satisfaction or regret following male-to-female sex reassignment surgery. Arch Sex Behav. 2003; 32(4):299-315.

19. Bodlund O, Armelius K. Self-image and personality traits in gender identity disorders: an empirical study. J Sex Marital Ther. 1994;20(4): 303-317.

20. Wolfradt U, Neumann K. Depersonalization, self-esteem and body image in male-to-female transsexuals compared to male and female controls. Arch Sex Behav. 2001;30(3):301-310.

21. Kraemer B, Delsignore A, Schnyder U, Hepp U. Body image and transsexualism. Psychopathology. 2008;41(2):96-100.

22. Weyers S, Elaut E, De Sutter P, et al. Long-term assessment of the physical, mental, and sexual health among transsexual women. $J$ Sex Med. 2009;6(3):752-760.

23. Rakic Z, Starcevic V, Maric J, Kelin K. The outcome of sex reassignment surgery in Belgrade: 32 patients of both sexes. Arch Sex Behav. 1996;25(5):515-525.

24. Newfield E, Hart S, Dibble S, Kohler L. Female-to-male transgender quality of life. Qual Life Res. 2006;15(9):1447-1457.

25. Kuhn A, Bodmer C, Stadlmayr W, Kuhn P, Mueller MD, Birkhäuser M. Quality of life 15 years after sex reassignment surgery for transsexualism. Fertil Steril. 2009;92(5):1685-1689.

26. Ainsworth TA, Spiegel JH. Quality of life of individuals with and without facial feminization surgery or gender reassignment surgery. Qual Life Res. 2010;19(7):1019-1024.

27. Murad MH, Elamin MB, Garcia MZ, et al. Hormonal therapy and sex reassignment: a systematic review and meta-analysis of quality of life and psychosocial outcomes. Clin Endocrinol (Oxf). 2010;72(2): 214-231.

28. Slabbekoorn D, van Goozen SH, Megens J, Gooren LJ, CohenKettenis PT. Activating effects of cross-sex hormones on cognitive functioning: a study of short-term and long-term hormone effects in transsexuals. Psychoneuroendocrinology. 1999;24(4):423-447.

29. Miles C, Green R, Hines M. Estrogen treatment effects on cognition, memory and mood in male-to-female transsexuals. Horm Behav. 2006; 50(5):708-717.

30. Leavitt F, Berger JC, Hoeppner JA, Northrop G. Presurgical adjustment in male transsexuals with and without hormonal treatment. J Nerv Ment Dis. 1980;168(11):693-697.

31. Slabbekoorn D, Goozen SHV, Gooren LJ, Cohen-Kettenis PT. Effects of cross-sex hormone treatment on emotionality in transsexuals. Int J Transgend. 2001;5(3):2-18.

32. Kuiper B, Cohen-Kettenis P. Sex reassignment surgery: a study of 141 Dutch transsexuals. Arch Sex Behav. 1988;17(5):439-457.

33. West S, King TS, Carey KN, et al. Systems to rate the strength of scientific evidence. Evid Rep Technol Assess (Summ). 2002;47:1-11.

34. Blanchard R, Clemmensen LH, Steiner BW. Gender reorientation and psychosocial adjustment in male-to-female transsexuals. Arch Sex Behav. 1983;12(6):503-509.
35. Gómez-Gil E, Zubiaurre-Elorza L, Esteva I, et al. Hormone-treated transsexuals report less social distress, anxiety and depression. Psychoneuroendocrinology. 2012;37(5):662-670.

36. Gorin-Lazard A, Baumstarck K, Boyer L, et al. Hormonal therapy is associated with better self-esteem, mood, and quality of life in transsexuals. J Nerv Ment Dis. 2013;201(11):996-1000.

37. Colizzi M, Costa R, Todarello O. Transsexual patients' psychiatric comorbidity and positive effect of cross-sex hormonal treatment on mental health: results from a longitudinal study. Psychoneuroendocrinology. 2014;39:65-73.

38. Bouman WP, Claes L, Marshall E, et al. Sociodemographic variables, clinical features, and the role of preassessment cross-sex hormones in older trans people. J Sex Med. 2016;13(4):711-719.

39. Fisher AD, Castellini G, Bandini E, et al. Cross-sex hormonal treatment and body uneasiness in individuals with gender dysphoria. J Sex Med. 2014;11(3):709-719.

40. Heylens G, Verroken C, De Cock S, T'Sjoen G, De Cuypere G. Effects of different steps in gender reassignment therapy on psychopathology: a prospective study of persons with a gender identity disorder. $J$ Sex Med. 2014;11(1):119-126.

41. Gómez-Gil E, Vidal-Hagemeijer A, Salamero M. MMPI-2 characteristics of transsexuals requesting sex reassignment: comparison of patients in prehormonal and presurgical phases. J Pers Assess. 2008;90(4): 368-374.

42. Bonierbale M, Baumstarck K, Maquigneau A, et al. MMPI-2 profile of French transsexuals: the role of sociodemographic and clinical factors. A cross-sectional design. Sci Rep. 2016;6:24281.

43. Gorin-Lazard A, Baumstarck K, Boyer L, et al. Is hormonal therapy associated with better quality of life in transsexuals? A cross sectional study. J Sex Med. 2012;9(2):531-541.

44. Gómez-Gil E, Zubiaurre-Elorza L, de Antonio IE, Guillamon A, Salamero M. Determinants of quality of life in Spanish transsexuals attending a gender unit before genital sex reassignment surgery. Qual Life Res. 2014;23(2):669-676.

45. Colizzi M, Costa R, Todarello O. Dissociative symptoms in individuals with gender dysphoria: is the elevated prevalence real? Psychiatry Res. 2015;226(1):173-180.

46. Asscheman H, Gooren LJ, Eklund PL. Mortality and morbidity in transsexual patients with cross-gender hormone treatment. Metabolism. 1989;38(9):869-873.

47. Su TP, Pagliaro M, Schmidt PJ, Pickar D, Wolkowitz O, Rubinow DR. Neuropsychiatric effects of anabolic steroids in male normal volunteers. JAMA. 1993;269(21):2760-2764.

48. King JA, Rosal MC, Ma Y, Reed G. Association of stress, hostility and plasma testosterone levels. Neuro Endocrinol Lett. 2005;26(4): 355-360.

49. Wylie K, Knudson G, Khan SI, Bonierbale M, Watanyusakul S, Baral S. Serving transgender people: clinical care considerations and service delivery models in transgender health. Lancet. Epub 2016 Jun 16.
Neuropsychiatric Disease and Treatment

\section{Publish your work in this journal}

Neuropsychiatric Disease and Treatment is an international, peerreviewed journal of clinical therapeutics and pharmacology focusing on concise rapid reporting of clinical or pre-clinical studies on a range of neuropsychiatric and neurological disorders. This journal is indexed on PubMed Central, the 'PsycINFO' database and CAS,
Dovepress

and is the official journal of The International Neuropsychiatric Association (INA). The manuscript management system is completely online and includes a very quick and fair peer-review system, which is all easy to use. Visit http://www.dovepress.com/testimonials.php to read real quotes from published authors. 